\title{
Meta
}

Journal des traducteurs

Translators' Journal

\section{JENN, Ronald et OSTER, Corine, dir. (2014) : Territoires de la traduction. Artois : Artois Presses Université, 212 p.}

\section{Oumarou Mal Mazou}

Volume 61, numéro 3, décembre 2016

URI : https://id.erudit.org/iderudit/1039235ar

DOI : https://doi.org/10.7202/1039235ar

Aller au sommaire du numéro

Éditeur(s)

Les Presses de l’Université de Montréal

ISSN

0026-0452 (imprimé)

1492-1421 (numérique)

Découvrir la revue

Citer ce compte rendu

Mal Mazou, O. (2016). Compte rendu de [JEnN, Ronald et Oster, Corine, dir. (2014) : Territoires de la traduction. Artois : Artois Presses Université, 212 p.]

Meta, 61(3), 748-750. https://doi.org/10.7202/1039235ar

Ce document est protégé par la loi sur le droit d'auteur. L'utilisation des services d'Érudit (y compris la reproduction) est assujettie à sa politique d'utilisation que vous pouvez consulter en ligne.

https://apropos.erudit.org/fr/usagers/politique-dutilisation/
Cet article est diffusé et préservé par Érudit.

Érudit est un consortium interuniversitaire sans but lucratif composé de l'Université de Montréal, l'Université Laval et l'Université du Québec à Montréal. Il a pour mission la promotion et la valorisation de la recherche. https://www.erudit.org/fr/ 
Lafarga, Francisco y Pegenaute, Luis, eds. (2013): Diccionario histórico de la traducción en Hispanoamérica. Madrid/Fráncfort: Iberoamericana/Vervuert.

Jenn, Ronald et Oster, Corine, dir. (2014): Territoires de la traduction. Artois: Artois Presses Université, 212 p.

Territoires de la traduction est un voyage dans l'espace et dans le temps, dans l'histoire et dans la géographie, dans la littérature et dans la traductologie. De la Renaissance au monde moderne, d'Italie en Allemagne, en passant par la France, les limites des "territoires» étudiés demeurent toujours instables et dynamiques. Un voyage magique avec pour point de départ la Renaissance mais comme point de chute le XxI siècle; une aventure traductologique qui regroupe dix contributions savamment compilées par Roland Jenn et Corinne Oster.

Les directeurs de cet ouvrage dont l'objectif est d'établir le rapport entre la traduction et le territoire sont tous deux enseignants de traduction et de traductologie à l'Université Charles-de-Gaulle de Lille. Jenn s'intéresse à la pseudo-traduction et aux rapports entre idéologie et traduction, notamment dans les échanges entre la France et les États-Unis. Oster, elle, est agrégée d'anglais et ses domaines de recherche incluent notamment les femmes en traduction, le cinéma féminin et le cinéma de la marge.

L'ouvrage commence par une introduction digeste, brève et concise, dans laquelle les compilateurs lillois définissent ce qu'ils entendent par territoire, c'est-à-dire «un espace pensé, approprié et imaginé par l'homme» (p. 7). Ensuite, ils établissent le rapport qui existe entre territoire et traductologie. Jenn et Oster, en observant la traduction, déduisent que celle-ci est une confirmation que le texte lui-même est un territoire avec ses caractéristiques propres. De même, la traductologie, en tant qu'espace théorique de la traduction, affirme sa territorialité à travers un ensemble de «provinces» que les différents contributeurs réunis dans cet ouvrage explorent de fond en comble.

Partrizia de Capitani ouvre la voie et se penche sur la traduction en français de nouvelles et de comédies italiennes du XVI ${ }^{e}$ siècle, plus particulièrement la traduction des toponymes. L'auteure étudie les stratégies de traduction qui dépendent surtout du type de réception. De l'analyse, il ressort que les traducteurs de nouvelles respectent généralement la localisation du texte de départ en traduisant les noms des lieux en français, ou en les reportant tels quels. Les traducteurs de comédies quant à eux ont tendance à effacer la provenance italienne des toponymes dans le texte traduit: «[le traducteur] revendique son effort pour ancrer la comédie italienne dans le territoire littéraire français» (p. 31). Capitani pense que le moyen le plus sûr de faciliter la compréhension entre territoires linguistiques différents, c'est de rendre le texte étranger familier en réduisant les différences (p. 32).

Mathilde Aubague consacre son étude à la traduction-adaptation de Guzmán de Alfarache d'Alemán faite par Albertinus. Elle montre que le système littéraire allemand n'est pas favorable à la réception du genre picaresque, pourtant en plein essor en Espagne. D'après l'auteure, le traducteur choisit la liberté en donnant de l'importance aux enjeux idéologiques, notamment le genre littéraire, la religion, la politique, l'espace géographique et l'aspect linguistique: "Albertinus, en publiant sa traduction, propose au public germanique un genre nouveau, mais qui est adapté aux conditions politiques et religieuses du Saint Empire du début du XviI iè siele» (p. 41). Dans son analyse, Aubague constate que le traducteur donne une importance particulière à l'aspect religieux. En définitive, la traduction d'Albertinus vise à susciter auprès du public germanophone une adhésion à la foi chrétienne.

Samuel Trainor, dans un article rédigé en anglais, plonge dans le $\mathrm{xIX}^{\mathrm{e}}$ siècle et propose une étude sur le rapport entre la langue nationale et les patois régionaux dans les écoles en France sous la III République. La politique de "l’aménagement du territoire» de cette époque procéderait, selon l'auteur, du défrichement linguistique à travers les exercices de traduction entre les patois et le français pour renforcer l'efficacité de la didactique normative. Faisant appel à un champ lexical agricole, il construit son analyse autour d'une métaphore champêtre, et sur les mots défrichement/ déchiffrement, ou encore dépaysement/repaysement pour décrire la politique linguistique imposée aux provinces françaises du xIX ${ }^{e}$ siècle. Pour lui, «translation came to be seen as a metaphor for transformative culture and as a vital means for its propagation» (p. 67). Là encore, la traduction assure son rôle d'instrument politique.

Caroline Marie examine quant à elle la difficulté à établir les contours d'un texte source ayant plusieurs versions comme c'est le cas de Mrs Dalloway, un texte de Virginia Woolf, où le traducteur est obligé de prendre position entre plusieurs textes « originaux». Outre cette difficulté, l'auteure montre que les traductions en français d'un texte comme celui de Woolf impliquent l'altérité qui risque de brouiller les traces de l'exotisme original voulu par l'auteure. Et à Marie de conclure: «La traduction de Frédéric Armelm comme les illustrations de Napom ne correspondent pas à un texte, mais à une constellation des textes palimpsestueux $[\ldots] »($ p. 93). 
Le brouillage des frontières entre le texte source et la traduction est également étudié par Dirk Weissmann dans la perspective de l'autotraduction. Envisagée «comme un cas de l'écriture plurilingue» chez certains et "pratique faisant suite à l'adoption d'une nouvelle langue d'écriture» (p. 98) chez d'autres, l'autotraduction, d'après Wessmann, «confirme le fonctionnement de la traduction comme frontière, tout en faisant entrevoir un autre mode d'organisation de l'espace littéraire» (p. 97). L’universitaire de Paris-Est Créteil dresse dans son article un aperçu historique de l'autotraduction littéraire et propose des éléments théoriques en prenant appui sur l'écriture trilingue de Samuel Becket, l'auteur et auto-traducteur de la célèbre pièce du théâtre de l'absurde En attendant Godot. Weissmann considère Becket comme un écrivain trilingue dont «l'écriture inclut un troisième pôle dans le processus création-traduction [...] représenté par la culture et la langue allemandes» (p. 103).

Parlant du plurilinguisme justement, Miriam Suchet s'appuie sur le roman The Voice du Nigérian Okara pour démontrer que le monolinguisme apparent de l'original peut cacher des traces d'autres langues et, par conséquent, l'original luimême n'est en quelque sorte qu'une traduction. Dans une étude approfondie de la préposition «inside» dont l'utilisation est abondante dans le roman, Suchet examine comment Jean Sévry, traducteur de The Voice en français, l'a traitée dans sa version française La Voix. Elle observe, à travers des exemples concrets, que le traitement de cette préposition polyphonique dans la traduction "permet de rendre la langue cible légèrement étrangère» (p. 120). La traduction, conclut-elle, «ne peut se réduire à l'image d'un pont qui relierait des territoires homogènes et clos sur eux-mêmes » (p. 123).

Simos Grammenidis, quant à elle, étudie l'impact des territoires sur les choix opérés par les différents traducteurs du roman Les particules élémentaires de Michel Houellebecq en grec, turc et anglais. L'auteur identifie à cet effet deux types de territoires: les territoires centraux et les territoires périphériques. D’après Grammenidis, en laissant «de la place pour que «l'Autre puisse se manifester sans pour autant négliger le Même» (p.138), le traducteur est influencé par son propre territoire culturel et celui-ci conditionne son attitude envers un texte étranger.

Dans un voyage métaphorique, Alessandra Risso crée "un lieu de convergence» entre les théories de la traduction, le postcolonialisme et la migration. S'appuyant sur une pléthore d'auteurs parmi les critiques, les philosophes et les traductologues entre autres, Risso analyse les modalités qui permettent à l'espace d'être un lieu dynamique pour les migrants et leurs diverses identités. Dans leur va-et-vient permanent, les cultures postcoloniales participent à la construction et à la déconstruction des espaces qu'elles traversent par le truchement de la traduction, en ce sens que celle-ci permet de transposer la culture, la langue et l'idée d'un espace vers un autre. L'argumentaire fort convaincant de l'auteure prend appui sur deux romans d'une écrivaine africaine, elle-même partagée entre deux territoires (réels) : l'Égypte et le Soudan. Chez Aboulela, "the concept of place emerges as physical entity located in-between translation and migration" (p. 143-144). Dans cette entité qui sert de territoire commun à la traduction et à la migration, la traduction se dote d'un pouvoir qui navigue entre les frontières linguistiques et culturelles et se donne le droit non seulement de sélectionner ce qui est important à ses yeux, mais aussi d'omettre ce qu'elle considère comme négligeable (p. 145).

Freddie Plassard de son côté scrute la traduction sous le prisme épistémologique et émet l'hypothèse selon laquelle la traductologie en elle-même est un territoire. Dans une perspective historique, Plassart, à la suite de Ballard (1995) et autres historiens de la traduction, perçoit la traductologie comme "une succession de tournants [...] ayant pour objet de déterminer où se situe le commencement de la réflexion sur la traduction» (p. 160). D'un point de vue discursif, elle dresse un état des lieux de la typologie des discours sur la traduction en revisitant les travaux des traductologues de renom tels que Berman, Mounin, Nida ou encore Ladmiral. D'autres perspectives sont également explorées et discutées, notamment les approches méthodologique et métalinguistique. Tout compte fait, Plassard pense que « toute forme de représentation est nécessairement réductrice par rapport à la réalité» et qu'elle «ne saurait par ailleurs être la seule voie ni dispenser d'un discours explicatif» (p. 169).

Enfin, Christine Raguet referme ce voyage à travers les territoires (physiques et symboliques) avec une sorte de (re)configuration des territoires traductifs. Selon elle, au lieu de se constituer un autre territoire, le texte traduit est lié à l'original par la distance parcourue qui, malgré les apparences, ne l'en éloigne pas. Au contraire, cette relation entre l'Un et l'Autre permet aux deux textes de créer un «tout-mosaïque, formé d'une multiplicité de tesselles» (p. 187).

On peut reprocher aux auteurs de l'ouvrage de n'avoir pas ouvert les frontières de leurs territoires traductologiques aux études asiatiques ou latino-américaines qui sont pourtant si vastes et en pleine expansion, mais ce serait trop leur demander dans un seul ouvrage. Une chose est sûre cependant, Territoires de la traduction apporte 
sa contribution au champ traductologique qui ne cesse de s'élargir et de s'affirmer comme une discipline autonome, même si elle ne peut se défaire complètement des autres disciplines avec lesquelles elle évolue depuis toujours: histoire, linguistique, littérature, philosophie et sciences pures.

Oumarou Mal Mazou

Université de Liège, Liège, Belgique

García, Adolfo M. y Arrizabalaga María Inés, eds. (2013): La traducción bajo la línea de la convergencia. Córdoba: Universidad Nacional de Córdoba, 283 p.

Esta obra reúne catorce trabajos cuya equilibrada distribución en cuatro partes, a saber: "La traducción como proceso (inter)cultural", "La traducción como actividad literaria”, "Traductología cognitiva" y "Estudios contrastivos", pone en manos del lector interesado el resultado de diversas investigaciones llevadas a cabo por los autores y que presentaron durante las III Jornadas Internacionales de Traductología, organizadas por el área de Traductología del Centro de Investigaciones de la Facultad de Lenguas de la Universidad Nacional de Córdoba (Argentina). Los catorce artículos van precedidos de un exordio, a manera de editorial, firmado por Adolfo M. García y María Inés Arrizabalaga: "La Traductología, una zona de contacto disciplinar", donde se exponen las líneas directrices sobre la interdisciplinareidad que converge en la traductología y, por ende, en la traductografía que enriquece las culturas meta y nos pone en antecedentes de los artículos -tratados como capítulos correlativos- que vamos a encontrar a lo largo de las páginas del volumen.

La primera parte se compone de tres artículos o capítulos. El primero de ellos, "Supuesto de equivalencia. La traducción cultural en el Perú» lo firma Aymará del Llano y versa sobre la traducción cultural en la literatura peruana del siglo XX, teniendo en cuenta la presencia de quechuismos en los textos literarios. En este punto, resulta inevitable recordar la dificultad de interpretar como lectora no iniciada en la lengua y cultura quechuas los magistrales textos de Arguedas, a título de ejemplo, y el imaginar los escollos con los que se encontraría un traductor a la hora de verter un texto arguediano ${ }^{1}$. Ante las dificultades que se le plantean al traductor al verter la cultura oral quechua a la cultura occidental americana del siglo $\mathrm{XX}$, la autora defiende la decisión de la supuesta falta de adecuación para que el lector sea consciente de la alteridad. Para introducirse en el estudio, la autora hace un recorrido histórico por el proceso de ida y vuelta que comenzó con la transcripción de la oralidad hasta la interpretación de la oralidad a través del texto escrito, tal y como sucede en la traducción de textos del género dramático. Al resultado del proceso de traducción cultural lo califica de efecto de traducción y dicho proceso en el que el traductor tiene en cuenta la alteridad permite al lector llegar a la identidad cultural presente en el texto.

La segunda aportación, «Hacia un concepto de traducción política. 1492/1992: El lenguaje y la memoria en América Latina», se debe a Ana Inés Leunda, quien aborda el tema desde la Semiótica de la Cultura y concretamente desde las ideas de Iuri Lotman. La autora analiza la inmersión en el contexto cultural en el que debe investigar y moverse el traductor, y cómo influye en el proceso translatorio y producto traductográfico la vivencia del contexto sociocultural por parte del traductor. Parte de la elaboración de los primeros diccionarios bilingües de la América hispana, el del franciscano fray Alonso de Molina para el par de lenguas náhuatlespañol, y el del dominico fray Domingo de Santo Tomás para el par quechua-español, ofreciendo algunos ejemplos de cómo el contexto cronoespacial, religioso, político y cultural pudo influir en la comprensión de términos y conceptos y en la elaboración de las entradas de dichos diccionarios.

El tercer capítulo, «Explosión y memes en la literaturización: ¿Problemas comunes en La saga de los confines y el Kalevala?», lo firma María Inés Arrizabalaga, coeditora del presente volumen. Aborda unos textos construidos como un rompecabezas de microrrelatos, de carácter fragmentario con un hilo conductor común y que comparten rasgos formales propios de la «creación del artefacto literario». Los límites del puzzle literario los fijan los llamados relatos marco, nexo entre los microrrelatos. A pesar de los opuestos geográficos, pues el primero de ellos se enmarca en el contexto espacial del Cono Sur, mientras que el segundo se desarrolla en el más extremo septentrión, en la región finlandesa de Carelia Occidental, ambos narran hechos enmarcados en la antropología, la etnohistoria y la literatura épica. En los dos es relevante la figura del narrador, que en el primero se trata de un escriba y traductor, y en el segundo de un bardo o cantor, que escriben, narran e interpretan unos hechos. Lo que nos resulta más interesante de este enjundioso artículo de Arrizabalaga es el hecho del rastreo que el investigador en historia de la traducción puede hacer en la literatura para sacar a la luz apuntes para una historia de los traductores y de cómo el oficio de traductor y/o intérprete se hace presente como personaje literario.

La segunda parte del volumen, «La traducción como actividad literaria», se abre con el artículo de Mercedes Rodríguez Temperley «Las 